\title{
O Papel da Home Literacy e da Educação Infantil no Desenvolvimento dos Precursores da Alfabetização
}

\section{The Role of Home Literacy and Early Childhood Education in the Development of Literacy Precursors}

\section{El Papel de la Alfabetización en el Hogar y la Educación Infantil Temprana en el Desarrollo de Precursores de Alfabetización}

\author{
Katia de Matos Palinha* \\ Universidade Salgado de Oliveira - Universo, Niterói, Rio de Janeiro, Brasil \\ Márcia Maria Peruzzi Elia da Mota** \\ Universidade Salgado de Oliveira - Universo, Niterói, Rio de Janeiro, Brasil
}

\begin{abstract}
RESUMO
Neste estudo, investigou-se o papel do Home Literacy Environment e da Educação Infantil no desenvolvimento dos precursores da alfabetização: consciência fonológica e conhecimento de letras. Participaram da pesquisa 53 estudantes do $1^{0}$ ano de escolas municipais de nível socioeconômico baixo e seus cuidadores, sendo analisadas: 1) as relações entre Home Literacy Environment e os precursores, por meio de correlações de Pearson, as quais não foram significativas; 2) a contribuição da Educação Infantil no desenvolvimento dos precursores, medida por meio de Testes $t$, comparando-se as diferenças entre os grupos 0 (pouca experiência) e 1 (com experiência em Educação Infantil), onde se obteve correlações significativas entre o conhecimento das letras e Consciência Fonológica. Esses dados sugerem que o Home Literacy Environment dessas crianças pode ser insuficiente para o desenvolvimento desses preditores, e, portanto, cursar a Educação Infantil influencia a alfabetização.
\end{abstract}

Palavras-chave: home literacy, alfabetização, educação infantil.

\begin{abstract}
In this study the role of Home Literacy Environment and Childhood Education in the development of literacy precursors was investigated: Phonological Awareness and knowledge of letters. 53 students of the $1^{\text {st }}$ Year of municipal schools of low socioeconomic level and their caregivers took part in he research, there were analyzed: 1) the relation between Home Literacy Environment and the precursors through Pearson correlations; 2) the contribution of Childhood Education to the development of literacy precursors. The results of mean's comparisons with t-test shows significant differences between groups with little experience and with experience in Preschool Child Education for Phonological Awareness and letter knowledge. Pearson correlations were not significant. The data analysis suggests that environment of these children may be insufficient for the development of
\end{abstract}


these predictors, and therefore attending Early Childhood Education influences literacy.

Keywords: home literacy, literacy, childhood education.

\section{RESUMEN}

Este estudio investigó el papel del Home Literacy Environment y la educación de la primera infancia en el desarrollo de los precursores de la alfabetización: Consciencia Fonological y el conocimiento de las letras. Participaron en la investigación, 53 estudiantes del primero año de escuelas municipales de bajo nivel socioeconómico y sus cuidadores, siendo analizados: : 1) las relaciones entre del Home Literacy Environment y los precursores, por medio de correlaciones de Pearson, el que no eran significativas; 2) la contribución de la educación de la primera infancia en el desarrollo de precursores, medido a través de pruebas $t$, comparando las diferencias entre los grupos 0 (poca experiencia) y 1 (con experiencia en educación infantil), donde obtuvimos correlaciones significativas entre el Conocimiento de las letras y la conciencia fonológica. Estos datos sugieren que el ambiente de alfabetización en la casa (Home Literacy) de estos niños puede ser insuficiente para el desarrollo de estos predictores, y por lo tanto para asistir a la educación de la primera infancia influye en la alfabetización.

Palabras clave: home literacy, alfabetismo, La educación de la primera infancia.

A escola e a família são as duas principais instituições de formação das crianças nos anos iniciais. As experiências familiares e escolares podem ser responsáveis pelas diferenças no desempenho acadêmico da criança (Niklas \& Schneider, 2013; Sénéchal \& LeFevre, 2014). Um aspecto das experiências que a criança tem em casa que tem sido investigado pela literatura internacional como sendo importante para aquisição da leitura e escrita é o Home Literacy Environment (HLE). Recentemente, Hamilton, Hayiou-Thomas, Hulme e Snowling (2016) caracterizaram HLE como um termo guarda-chuva, que se refere às interações relacionadas às experiências, aos recursos e às atitudes em relação à alfabetização que a criança tem em casa. No Brasil, Mota (2014) definiu esse termo como as experiências que a criança tem em casa com a língua escrita. Essas experiências podem ser promovidas pelos pais ou por um cuidador, mas têm como característica fazer parte da vida doméstica da criança.

O interesse no estudo do Home Literacy Environment (HLE) vem de pesquisas que mostram que medidas de HLE predizem o sucesso na alfabetização. Sénéchal, LeFevre, Thomas e Daley (1998), por exemplo, investigaram o papel do HLE em precursores da alfabetização. Nesse estudo, o HLE foi medido como exposição a livros de histórias infantis. Entre os precursores da alfabetização, os autores focaram na avaliação do vocabulário e da consciência fonológica. Essas habilidades contribuíram para alfabetização. Dockerell, Stuart e King (2008) mostraram que desenvolver o 
vocabulário de crianças em educação infantil contribuiu para alfabetização dessas crianças.

A consciência fonológica, a habilidade de refletir sobre os sons da fala, é outro importante preditor da aquisição de leitura e escrita das crianças (Capovilla \& Capovilla, 2000; Cardoso-Martins, 1991). Em um estudo, Sénéchal et al. (1998) observaram que a exposição aos livros de histórias infantis (HLE) predisse o aumento do vocabulário e da consciência fonológica dessas crianças. Em um estudo subsequente, Sénéchal e LeFevre (2002) mostraram que a exposição ao livro de histórias foi preditor do desenvolvimento do vocabulário infantil, mas não da consciência fonológica. Os resultados contraditórios encontrados pela autora levaram Sénéchal (2006) a continuar avaliando o papel que o HLE exerce nos precursores da alfabetização. Em um estudo longitudinal no qual incluiu variáveis de escrita e duas medidas de HLE (leitura de livros e ensino dos pais), os resultados observados mostraram que a leitura de livros tinha relação direta com as habilidades de linguagem infantil, como o vocabulário, mas não se mostrou estar relacionada à alfabetização precoce da criança ou à consciência do fonema (um aspecto da consciência fonológica). Por outro lado, quando a medida de HLE foi o ensino dos pais sobre a alfabetização, foi observada uma relação direta com as habilidades de alfabetização emergente das crianças e indireta com a consciência do fonema.

Esses estudos chamam atenção para a razão pela qual Hamilton et al. (2016) definem o HLE como um constructo guarda-chuva. A forma com que os pais lidam com a escrita em casa difere, sendo que algumas interações podem ser mais formais (ensino de letras) e outras menos formais - leitura compartilhada (Sénéchal et al., 1998) e, como foi visto, essas parecem afetar de forma diferente 0 desempenho da escrita e de seus precursores.

No contexto nacional, Cardoso e Mota (2015) investigaram a relação entre um questionário mais amplo de HLE e precursores cognitivos da alfabetização. O questionário envolvia levantar informações sobre atividades formais e informais. Em relação aos precursores da alfabetização, as autoras avaliaram o vocabulário, conhecimento do nome das letras e consciência fonológica. Os resultados mostram correlações positivas e significativas entre os escores do questionário e os três precursores da alfabetização, indicando que as crianças com experiências mais ricas de HLE também tinham desempenho melhor nos precursores da alfabetização.

No presente estudo, avaliamos as experiências da criança com a escrita no ambiente de casa, com um questionário similar ao de Cardoso e Mota (2015). No entanto, investigamos também o papel da educação infantil no desenvolvimento desses precursores. Uma questão que pode ter influenciado o estudo de Cardoso e Mota (2015) 
era a experiência prévia que a criança tinha com a educação infantil. Essa experiência não foi controlada pelas autoras.

Paschoal e Machado (2009) destacaram que até meados da década de 1970, pouco se tinha caminhado com relação à criação de uma legislação que garantisse a oferta da Educação Infantil, sendo preciso quase um século para que a criança obtivesse seus direitos referentes à educação garantidos numa legislação. Hoje, acredita-se que a Educação Infantil tem como função primária fornecer subsídios que corroborem para o desenvolvimento biopsicossocial da criança por completo, por meio da participação de profissionais qualificados, buscando excelência no desenvolvimento infantil e prevenindo as possíveis dificuldades e defasagens na aprendizagem e na vida acadêmica futura. Com relação ao que compete à Educação infantil, é destacada a importância do trabalho preventivo na redução das dificuldades.

Segundo Mathias e Paula (2009), a incorporação da Educação Infantil, na LDB (Lei 9394/96), como etapa inicial da educação básica, poderá fazer com que esta modalidade de ensino perca um pouco do seu caráter assistencialista e passe a exercer duas funções: a de educar e a de cuidar. A primeira função relaciona-se com o fato de que cabe a Educação Infantil o papel de desenvolver as primeiras noções da linguagem escritas, estreitando o contato das crianças com a língua e fazendo com que essas sejam capazes de criar estratégias e habilidades que as façam refletir sobre a linguagem oral e adquiram pistas iniciais que podem Ihes auxiliar a refletir sobre a construção da linguagem escrita. Dessa forma, a Educação Infantil pode vir a oferecer experiências com a leitura e escrita que as crianças precisam para se alfabetizar. É possível que em casos em que a criança tenha menos experiências em casa, a escola faça diferença fundamental no desenvolvimento da alfabetização.

O objetivo do presente estudo é avaliar a influência de fatores ambientais como as práticas de HLE em precursores da alfabetização em crianças de escolas públicas brasileiras. Adicionalmente, investigamos o papel que a participação na Educação Infantil exerce no desenvolvimento de precursores da alfabetização: consciência fonológica, vocabulário e conhecimento do nome das letras.

\section{Método}

Participaram do estudo 53 crianças, com idade média de 6,92 anos $(\mathrm{DP}=0,68)$, estudantes do $1^{\circ}$ ano do Ensino Fundamental de escolas da Rede Pública de Ensino, localizadas nos municípios de Itaboraí, Nova Iguaçu e São Gonçalo no estado do Rio de Janeiro, que atendem a uma clientela de classe popular. Também participaram os pais das crianças que responderam ao Questionário Home Literacy e 
Dados Sociodemográficos. Este estudo foi aprovado pelo Comitê de Ética em Pesquisa da Universidade dos autores. O critério de inclusão no estudo foi a assinatura do termo de Consentimento Livre e Esclarecido, pelos responsáveis. A participação das crianças ocorreu por meio de contato pessoal, sendo o convite feito individualmente. Dessa forma, a participação na pesquisa foi voluntária, mediante consentimento dos participantes. A média de renda familiar foi 1,13 salários mínimos.

\section{Instrumentos}

\section{Questionário de "Home Literacy" e Dados Sociodemográficos}

Trata-se da adaptação do Questionário inicialmente elaborado por Chaney (1994), que foi adaptado por Mota (1998) para realidade brasileira. O questionário é composto por 17 itens, sendo que alguns permitem pontuações que variavam, por exemplo, de 0 a 5 . Por exemplo, para pergunta "Quem tem hábito de leitura em sua casa. Dessa forma, a pontuação máxima do questionário é de 115 pontos. A segunda parte do questionário refere-se aos dados de natureza sociodemográfica das famílias envolvidas e foi analisado separadamente.

\section{CONFIAS - Consciência Fonológica Instrumento de Avaliação Sequencial}

O CONFIAS (Moojen et al., 2003) é um instrumento cujo objetivo é medir habilidades ligadas ao desenvolvimento da Consciência Fonológica em crianças não alfabetizadas ou em processo de alfabetização. É composto por tarefas de síntese, segmentação, identificação, produção, exclusão e transposição silábica e fonêmica. Avalia diferentes níveis de desenvolvimento da consciência fonológica. O instrumento é dividido em duas partes, sendo que a primeira corresponde à consciência da sílaba e é composta pelos seguintes itens: síntese (S1), segmentação (S2), identificação da sílaba inicial (S3), identificação de rima (S4); produção de palavra com a sílaba dada (S5), identificação da sílaba medial (S6), produção de rima (S7), exclusão (S8) e transposição (S9). A segunda parte refere-se à consciência do fonema, sendo composta por: produção de palavra que inicia com o som dado ( $F 1)$, identificação de fonema inicial (F2), identificação de fonema final (F3), exclusão (F4), síntese (F5), segmentação (F6), transposição (F7). Foram utilizadas todas as tarefas, sendo 40 questões referentes à consciência da sílaba e 30 questões de consciência do fonema, totalizando 70 questões, cuja 
pontuação é definida por 1 ponto para cada resposta correta e 0 para cada resposta incorreta.

\section{Teste de Conhecimento do Nome das Letras}

A tarefa de conhecimento do nome das letras aplicada nesse estudo foi similar à de Barrera e Santos (2016). Foi apresentada à criança um cartão com as letras do alfabeto dispostas em ordem aleatória e pedido à criança a nomeação de cada uma delas. As letras foram impressas em maiúscula, em formato bastão. Todas as 26 letras do alfabeto foram apresentadas às crianças e atribuído 1 (um) ponto para cada resposta correta, e 0 (zero) para as respostas incorretas, totalizando a nota máxima de 26 (vinte e seis) pontos.

\section{Procedimentos}

Primeiramente foi solicitada a autorização nas escolas para realização da pesquisa. O trabalho foi realizado em duas etapas. Na primeira etapa, apresentou-se o Termo de Consentimento Livre e Esclarecido, juntamente com o Questionário de Home Literacy e dados Sociodemográficos, aos responsáveis dos alunos, de preferência o cuidador principal. Na segunda parte, foram realizadas as entrevistas com as crianças de forma individual, em uma sessão de cerca de 30 minutos, nas quais foram aplicados os instrumentos destinados à criança. Para o CONFIAS, se seguiu as instruções do manual. As letras foram apresentadas de forma aleatória e pediu-se à criança que as identificasse.

\section{Resultados}

Antes de iniciar a análise dos dados, foi verificado e confirmado a normalidade das variáveis para a realização das análises paramétricas. A Tabela 1 mostra a média, o desvio padrão, assimetria e curtose de cada variável. Seguindo os valores recomendados de assimetria e curtose de Tabachnick e Fidel (2007), sendo a assimetria dividida pelo erro padrão da assimetria não pode passar de dois, todas as variáveis foram adequadas a análises paramétricas. 
Tabela 1

Dados Descritivos para as Variáveis do Estudo

\begin{tabular}{lll}
\hline Variáveis & & \\
\hline CONFIAS_SILABAS & Média & 21,53 \\
& Desvio Padrão & 7,00 \\
& Assimetria & 0,04 \\
& Curtose & $-0,49$ \\
CONFIAS_FONEMAS & Média & 8,81 \\
& Desvio Padrão & 4,71 \\
& Assimetria & 0,53 \\
CONFIAS_TOTAL_ & Curtose & $-0,45$ \\
& Média & 30,34 \\
& Desvio Padrão & 11,13 \\
HL_TOT & Assimetria & 0,31 \\
& Curtose & $-0,60$ \\
& Média & 48,35 \\
& Desvio Padrão & 13,71 \\
CONHEC_NOME_LETRAS_- & Assimetria & $-0,37$ \\
TOTAL & Curtose & $-0,26$ \\
& Média & 13,86 \\
& Desvio Padrão & 8,60 \\
& Assimetria & $-0,04$ \\
& Curtose & $-1,37$ \\
\hline
\end{tabular}

Para responder à primeira pergunta de pesquisa que fizemos: "Há relação entre HLE e o desenvolvimento dos precursores da alfabetização?" foram realizadas correlações de Pearson entre os escores do Questionário de Home Literacy Environment e os precursores da alfabetização (consciência fonológica e conhecimento do nome de letras) que mostram resultados estatisticamente não significativos, conforme a Tabela 2 . No entanto, correlações significativas positivas moderadas ou fortes são encontradas entre 0 conhecimento do nome das letras e as tarefas de consciência fonológica. Esses resultados não eram os esperados pela hipótese formulada que sugeria que mais experiência com a leitura em casa (HLE) levaria a melhor desempenho nos precursores da alfabetização 
Tabela 2

Correlações de Pearson entre os escores de Home Literacy, Consciência Fonológica (Confias fonemas, Confias Silabas e Confias Total) e Conhecimento do nome das letras

\begin{tabular}{lccc}
\hline Variáveis & $\begin{array}{c}\text { CONFIAS } \\
\text { Fonemas }\end{array}$ & CONFIAS Total & $\begin{array}{c}\text { Home Literacy } \\
\text { Total }\end{array}$ \\
\hline CONFIAS sílabas & $0,79^{* *}$ & $0,96^{* *}$ & 0,07 \\
CONFIAS fonemas & & $0,92^{* *}$ & 0,03 \\
CONFIAS total & & 0,06 \\
Home Literacy Total & & \\
\hline Nota. ${ }^{* *} \mathrm{p}<0,01$ & & &
\end{tabular}

A segunda pergunta feita foi se "A Educação Infantil contribui para o desenvolvimento dos precursores da alfabetização?". Essa pergunta foi analisada por meio de Testes $t$, cujo objetivo foi verificar se as variáveis dependentes (Consciência Fonológica e Conhecimento do Nome das Letras) diferem-se em relação a dois grupos formados: 0 primeiro grupo, denominado grupo com baixa experiência, teve pouca (até um ano de experiência) ou nenhuma experiência com a Educação Infantil e o segundo grupo, com experiência com Educação Infantil, foi formado por crianças com um ou mais anos de educação infantil. Assim, o grupo com baixa experiência (BE) $(n=20)$ correspondeu às crianças que não frequentaram as classes de Educação Infantil ou que frequentaram por menos de um ano, enquanto o grupo com experiência, denominado grupo com alta experiência (AE) $(n=33)$ que correspondeu a crianças que frequentaram mais de um ano de Educação Infantil.

Os desempenhos das crianças dos dois grupos foram comparados, em relação aos escores médios obtidos nos testes de conhecimento de letras e consciência fonológica, por meio do Teste $t$ para grupos independentes. No caso da consciência fonológica, testes independentes foram feitos para cada um dos seguintes testes: Confias_Sílabas, Confias_Fonemas e Confias_Total. Avaliou-se também experiências no Home Literacy Environment. A Tabela 3 mostra as médias, desvio padrão e erro padrão para os grupos $B E$ e $\mathrm{AE}$, para cada uma das variáveis. 


\begin{tabular}{llllc}
$\begin{array}{l}\text { Tabela } 3 \\
\text { Comparativo entre grupos que cursaram ou não cursaram a Educação Infantil }\end{array}$ \\
\hline \multirow{2}{*}{ Variáveis } & GRUPO EI & $\mathrm{N}$ & MÉDIA & $\begin{array}{c}\text { DESVIO } \\
\text { PADRÃO }\end{array}$ \\
\hline CONFIAS SÍLABAS & 0 & 20 & 19,17 & 6,30 \\
& 1 & 33 & 22,96 & 7,11 \\
CONFIAS FONEMAS & 0 & 20 & 6,75 & 2,88 \\
& 1 & 33 & 10,06 & 5,19 \\
CONFIAS TOTAL & 0 & 20 & 25,92 & 8,47 \\
& 1 & 33 & 33,03 & 11,80 \\
HOME LITERACY TOTAL & 0 & 20 & 45,25 & 15,87 \\
& 1 & 33 & 50,24 & 12,10 \\
CONHECIMENTO DO NOME DE & 0 & 20 & 10,55 & 8,04 \\
LETRAS TOTAL & 1 & 33 & 15,87 & 8,40 \\
\hline
\end{tabular}

As médias do grupo $A E$ foram superiores às do grupo $B E$ para todas as variáveis analisadas, ainda que tais diferenças fossem discretas. Os resultados do Teste $t$ mostraram que frequentar a Educação Infantil gerou médias significativamente superiores para as variáveis: Confias Fonemas $\mathrm{t}_{(51)}=-2,98 ; p=0,004$; Confias Total $\mathrm{t}_{(51)}=-2,34 p=$ 0,02 e o Conhecimento do Nome das Letras $\mathrm{t}_{(51)}=-2,27 \quad p=0,02$. Apenas, para as variáveis Confias Sílabas $\mathrm{t}_{(51)}=-1,96 p=0,055$ e Home Literacy $\mathrm{t}_{(51)}=-1,29 \quad p=0,20$ os resultados foram não significativos.

\section{Discussão}

Pesquisas têm demonstrado que a família pode auxiliar no desenvolvimento de habilidades que facilitam a aquisição da língua escrita (Niklas \& Schneider, 2013; Sénéchal \& LeFevre, 2014). No presente estudo, procurou-se compreender a relação entre as práticas de alfabetização em casa (HLE) e da Educação Infantil, no desenvolvimento dos precursores cognitivos da leitura e da escrita. Os precursores estudados foram a consciência fonológica e conhecimento do nome das letras, uma vez que essas duas habilidades são importantes para leitura (Ehri, 2005). Para investigar as relações descritas anteriormente, foram feitas duas perguntas: na primeira, buscou-se analisar se havia relações entre as práticas de HLE e o desenvolvimento dos precursores da alfabetização; a segunda pergunta destinava-se a verificar se frequentar as classes de Educação Infantil contribui para o desenvolvimento dos precursores da alfabetização.

Estudos anteriores mostraram que um ambiente alfabetizador rico em recursos pode gerar resultados positivos para alfabetização. 
Esperava-se, então, no presente estudo, que as correlações entre os escores de HLE e os precursores da alfabetização fossem significativos. Isto é, as crianças com os melhores resultados nas tarefas precursoras da alfabetização, fossem as que tivessem escores mais altos no questionário de HLE. As correlações, porém, para esse estudo não foram significativas. Esse foi um resultado inesperado e é necessário se pensar as razões para tal.

Afirmamos anteriormente que é esperado que um ambiente rico em experiências com a leitura e escrita prediga sucesso na alfabetização. Nosso questionário avaliou diferentes aspectos das experiências de leitura das crianças, sendo que a pontuação máxima do questionário era de 115 pontos. Ao analisarmos as características da amostra estudada, vimos que as crianças que participaram deste estudo eram oriundas de classes sociais baixas. Diferentes tipos de famílias podem fornecer recursos e experiências distintas para seus filhos (Chiu \& Mc Bride-Chang, 2010). Por exemplo, Strasser e Lissi (2009) apontam que em países em desenvolvimento as condições econômicas da família podem dificultar o acesso aos livros. Além disso, o baixo nível de escolaridade dos pais entrevistados aqui pode ter menos experiência com a escrita, que por sua vez acaba por não ter impacto na aquisição de precursores da alfabetização.

$\mathrm{Na}$ presente amostra, a renda média da família foi cerca de um salário mínimo. Os cuidadores das crianças também tinham baixa instrução, sendo que mais da metade da amostra desse estudo (cerca de $54,7 \%$ ) não tinha o Ensino Médio completo e cerca de $86,8 \%$ dos cuidadores não tinham o Ensino Superior. Dessa forma, as práticas de HLE desenvolvidas pela família nesse contexto podem não ter sido suficientes para o desenvolvimento de preditores da alfabetização. Se olharmos a média dos escores no questionário de HLE, veremos que, de fato, ela foi baixa, ou seja, menos de $50 \%$ do total possível de pontos (48 em 115 pontos). Esse conjunto de resultados sugere que a falta de correlações significativas entre os precursores da alfabetização e os escores de HLE pode ser atribuída a ambientes familiares que não chegam a oferecer experiências suficientes para ajudar as crianças na educação formal.

Por outro lado, temos observado que a Educação Infantil tem ocupado um espaço cada vez mais importante no sentido de preocupar-se com assuntos relacionados à instrução (Mathias \& Paula, 2009). Nesse sentido, as práticas pedagógicas na educação infantil podem complementar as experiências da criança com atividades de linguagem oral e escrita necessárias para alfabetização. A análise dos resultados deste estudo indica que este é o caso. A Educação Infantil pode contribuir para o desenvolvimento dos preditores da leitura e da escrita. O grupo que teve mais de um ano de experiência na educação Infantil obteve melhor desempenho do que $o$ que não teve esta experiência, em quase todas as tarefas 
apresentadas. A única em que não houve diferença significativa entre os grupos foi na de sílabas. Cabe ressaltar que as tarefas de sílaba são muitas vezes apontadas na literatura como tarefas de percepção da linguagem e não tanto de consciência fonológica (Liberman, Shankweiler, Fisher, \& Carter, 1974), o fato de envolver a percepção da fala e não a reflexão sobre esta pode explicar porque os dois grupos não diferiram nessa tarefa. Nessa idade, é esperado que as crianças não tenham dificuldades em perceber a fala e quando a tarefa necessita de reflexão mais explícita sobre ela, as experiências na educação infantil parecem fazer a diferença.

De um modo geral, podemos dizer que os dados analisados neste estudo nos levam a pensar na importância da Educação Infantil, especialmente em áreas mais carentes do Brasil. Cursar as classes de Educação Infantil auxiliou no desenvolvimento de precursores da linguagem escrita no nosso estudo. Uma questão que precisa ainda ser respondida é se elas compensam a ausência dessas experiências em casa, quando a escolarização formal inicia. Avaliamos apenas os precursores da alfabetização e precisamos saber se essas experiências iniciais continuam favorecendo a aquisição da língua escrita quando a alfabetização formal se inicia. Estudos futuros devem responder essas perguntas usando delineamentos longitudinais.

Em suma, podemos perceber, diante dos nossos dados, que investir na ampliação da oferta de Educação Infantil pode ser uma boa estratégia para aumentar o nível de desempenho dessas crianças quando ingressam no Ensino Fundamental. Precisamos também intensificar as campanhas que visem o desenvolvimento de práticas de leitura e escrita em casa, pois essa parece ser uma variável importante para o desempenho das crianças.

O presente estudo apresenta algumas limitações. Uma limitação deste estudo foi o tamanho da amostra. A dificuldade de encontrar com os pais na escola, visto que muitas dessas crianças vão para o colégio com os irmãos mais velhos e outros cuidadores, dificultaram a aplicação de questionários. Outro obstáculo observado foi o de conseguir crianças que não haviam cursado as classes de Educação Infantil, para podermos comparar com o grupo que cursou. Novas leis que tornam a educação infantil obrigatória e 0 fato de que atualmente as crianças são inseridas em creches, algumas delas antes de completar um ano de idade, porque seus pais precisam trabalhar para garantir o sustento da família, tornam essa amostra difícil de acesso. Por fim, o questionário utilizado de HLE não teve suas características psicométricas avaliadas para amostras brasileiras, mas destaca-se que foi utilizado em estudos anteriores já citados com resultados estatisticamente significativos. 


\section{Considerações finais}

Ao analisar o cenário da Educação Brasileira, percebe-se certa preocupação sobre as questões que permeiam a aquisição da linguagem escrita, visto que esse momento pode sustentar as aprendizagens futuras e possibilitar o domínio da língua como forma de comunicação, compreensão, expressão e produção do pensamento humano, bem como a formação intelectual do indivíduo, ou seja, "o domínio dos atos de ler e escrever é considerado, e não sem a devida razão, a base essencial para o sucesso escolar e para as aprendizagens futuras" (Roazzi, Minervino, \& Melo, 2014).

Esta pesquisa pode vir a contribuir para pensarmos em práticas de intervenção visando a melhoria da alfabetização. Foi possível observar que investir na oferta da Educação Infantil pode preparar melhor estas crianças para a alfabetização, mas é preciso pensar também na natureza das atividades que a escola vai promover para, de forma mais eficaz, facilitar a aprendizagem da leitura e escrita.

\section{Referências}

Barrera, S. D., \& Santos, M. J. (2016). Conhecimento do nome das letras e habilidades iniciais em escrita. Boletim Academia Paulista de Psicologia, 36, 1-15. Recuperado de http://pepsic.bvsalud.org/scielo.php?script=sci_arttext\&pid=S1 415-711X2016000100002

Brasil. Ministério da Educação e Cultura. (1996). Lei de Diretrizes e Bases da Educação Nacional. Lei no 9394, de 20 de dezembro de 1996. Dispõe sobre as Diretrizes e Bases da Educação Nacional. Brasília, DF: MEC.

Capovilla, A. G. S., \& Capovilla, F. C. (2000). Efeitos do treino de consciência fonológica em crianças com baixo nível socioeconômico. Psicologia Reflexão e Crítica, 13(1), 7-24. doi:10.1590/S0102-79722000000100003

Cardoso, C. V., \& Mota, M. M. P. E. (2015). Home-Literacy e os precursores da alfabetização. Revista Estudos e Pesquisas em Psicologia, 15(2), 708-724. doi:http://pepsic.bvsalud.org/scielo.php?script=sci_abstract\&pi $\mathrm{d}=$ S1808-42812015000200015\&lng = pt\&nrm=iso

Cardoso-Martins, C. (1991). A consciência fonológica e a aprendizagem inicial da leitura e da escrita. Cadernos de Pesquisa, 76, 41-49. Recuperado de http://publicacoes.fcc.org.br/ojs/index.php/cp/article/view/105 $3 / 1061$

Chaney, C. (1994). Language development, metalinguistic awareness and emergent literacy skills of 3 years-old children in relation to 
social class. Applied Psycholinguistics, 15(3), 371-394. doi: $10.1017 /$ S0142716400004501

Chiu, M. M., \& McBride-Chang, C. (2010). Family and reading in 41 countries: Differences across cultures and students. Scientific Studies of Reading, 14(6), 514-543. doi: $10.1080 / 10888431003623520$

Dockrell, J., Stuart, M., \& King, D. (2008). Favorecendo a aquisição e o desenvolvimento da linguagem oral: Teoria e evidências empíricas. In L. R. Castro, \& V. Besset. (Orgs.), Pesquisaintervenção na infância e juventude (pp. 322-344). Rio de Janeiro: Trarepa/Faperj.

Ehri, L. C. (2005). Learning to read words: Theory, findings and issues. Scientific Studies of Reading, 9(2), 167-188. doi: $10.1207 /$ s1532799xssr0902_4

Hamilton, L. G., Hayiou-Thomas, M. E., Hulme, C., \& Snowling, M. J. (2016). The home literacy environment as a predictor of the early literacy development of children at family-risk of dislexia. Scientific Studies of Reading, 20(5), 401-416. doi: $10.1080 / 10888438.2016 .1213266$

Liberman, I. Y., Shankweiler, D. Fischer, F. W., \& Carter, B. J. (1974). Explicit syllable and phoneme segmentation in the young child. Journal of Experimental Child Psychology, 18(2), 201-212. doi:10.1016/0022-0965(74)90101-5

Mathias, E. C. B., \& Paula, S. N. (2009). Educação Infantil no Brasil: Avanços, desafios e políticas públicas. Revista interfaces: Ensino, pesquisa e extensa, 1(1), 13-16.

Moojen, S., Lamprecht, R., Santos, R. M., Freitas, G. M., Brodacz, R.; Siqueira, Costa, A. C., \& Guarda, E. (2003). Consciência fonológica: Instrumento de avaliação sequencial. São Paulo, SP: Casa do Psicólogo.

Mota, M. M. P. E. (1998). Facilitadores cognitivos da Alfabetização. Relatório Técnico-científico apresentado ao CNPq.

Mota, M. M. P. E. (2014). Home-literacy e alfabetização: Uma revisão sistemática da literatura. Revista Psicologia Argumento, 32(78), 109-115. doi:10.7213/psicol.argum.32.078.A006

Niklas, F., \& Schneider, W. (2013). Home literacy environment and the beginning of reading and writing. Contemporary Educational Psychology, 38(1), 40-50. doi:10.1016/j.cedpsych.2012.10.001

Paschoal, J. D., \& Machado, M. C. G. (2009). A História da educação infantil no Brasil: Avanços, retrocessos e desafios dessa modalidade educacional. Revista HISTERDBR On-line, 9(33), 78-86. doi:10.20396/rho.v9i33.8639555

Roazzi, A., Minervino, C. A. S. M., \& Melo, M. R. A. (2014). Aprendizagem da leitura e da escrita: Princípios teóricos, históricos, níveis conceituais e aspectos motivacionais do processo de aprendizagem do código alfabético. In A. Roazzi, F. 
V. Paula, \& M. J. Santos (Orgs.), Leitura \& Escrita: A sua Aprendizagem na Teoria e na Prática (pp. 19-40). Curitiba: Juruá.

Sénéchal, M. (2006). Testing the home literacy model: Parent involvement in kindergarten is differentially related to Grade 4 reading comprehension, fluency, spelling, and reading for pleasure. Scientific Studies of Reading, 10(1), 59-87. doi: $10.1207 /$ s1532799xssr1001_4

Sénéchal, M., LeFevre, J.-A., Thomas, E., \& Daley, K. (1998). Differential effects of home literacy experiences on the development of oral and written language. Reading Research Quarterly, 33(1), 96-116. doi:10.1598/RRQ.33.1.5

Sénéchal, M., \& LeFevre, J. (2002). Parental involvement in the development of children's reading skill: A 5-year longitudinal study. Child Development, 73(2), 445-460. doi:10.1111/14678624.00417

Sénéchal, M., \& LeFevre, J. (2014). Continuity and change in the home literacy environment as predictors of growth in vocabulary and reading. Child development, 85(4), 1552-1568. doi: $10.1111 /$ cdev. 12222

Strasser, K., \& Lissi, M.R. (2009). Home and instruction effects on emergent literacy in a sample of kindergarten Chilean children. Society for the Scientific Study of Reading, 13(2), 175-204. doi: $10.1080 / 10888430902769525$

Tabachnick, B., \& Fidell, L. (2007). Using Multivariate Statistics. Needham Heigts: Allyn e Bacon.

\section{Endereço para correspondência \\ Katia de Matos Palinha}

Rua Marechal Deodoro, 217, $2^{\circ}$ andar, Centro, CEP 24030-060, Niterói - RJ, Brasil Endereço eletrônico: katia.m.palinha@gmail.com

\section{Márcia Maria Peruzzi Elia da Mota}

Rua Marechal Deodoro, 217, $2^{\circ}$ andar, Centro, CEP 24030-060, Niterói - RJ, Brasil Endereço eletrônico: mmotapsi@gmail.com

Recebido em: 02/06/2019

Reformulado em: 20/09/2019

Aceito em: 02/10/2019

\section{Notas}

* Mestre em Psicologia pela Universidade Salgado de Oliveira.

** Doutora em Psicologia pela Universidade de Oxford, Bolsista de Produtividade em Pesquisa nível 2 CNPq, Cientista do Nosso Estado FAPERJ.

Este artigo de revista Estudos e Pesquisas em Psicologia é licenciado sob uma Licença Creative Commons Atribuição-Não Comercial 3.0 Não Adaptada. 